\title{
Impact of metabolic indices on central artery stiffness: independent association of insulin resistance and glucose with aortic pulse wave velocity
}

\author{
D. R. Webb • K. Khunti $\cdot$ R. Silverman • L. J. Gray • \\ B. Srinivasan $\cdot$ P. S. Lacy $\cdot$ B. Williams $\cdot$ M. J. Davies
}

Received: 7 October 2009 /Accepted: 19 January 2010/Published online: 6 March 2010

(C) Springer-Verlag 2010

\begin{abstract}
Aims/hypothesis Non-invasive measures of aortic stiffness reflect vascular senescence and predict outcome in diabetes. Glucose-mediated elastic artery sclerosis may play an integral role in the development of macrovascular complications. We used carotid-femoral pulse wave velocity ( ${ }_{\mathrm{cf}} \mathrm{PWV}$ ) to quantify independent associations of fasting glucose, post-challenge glucose and derived insulin resistance (HOMA-IR) with aortic stiffness.

Methods ${ }_{\mathrm{cf}} \mathrm{PWV}$ was measured using a $4 \mathrm{MHz}$ continuous wave Doppler ultrasound probe within groups with newly identified age- and sex-matched normal glucose metabolism (NGM), impaired glucose regulation (IGR) and diabetes mellitus populations $(n=570$, mean age 59.1, $56 \%$ male).

Results After multivariate adjustment, IGR and diabetes were associated with significant aortic stiffening compared with NGM (adjusted ${ }_{\mathrm{cf}} \mathrm{PWV} \pm \mathrm{SE}$ : NGM, $9.15 \pm 0.12 \mathrm{~m} / \mathrm{s}$; IGR $9.76 \pm 0.11 \mathrm{~m} / \mathrm{s}, p<0.001$; diabetes, $9.89 \pm 0.12 \mathrm{~m} / \mathrm{s}, p<$ $0.001)$. IGR stratification indicated that impaired fasting glucose (IFG; $9.71 \pm 0.12 \mathrm{~m} / \mathrm{s}$ ) and post-challenge (impaired glucose tolerance; $9.82 \pm 0.24 \mathrm{~m} / \mathrm{s}$ ) categories had similar ${ }_{c f} \mathrm{PWV}(p=0.83)$. Modelled predictors of ${ }_{\text {cf }} \mathrm{PWV}$ were used
\end{abstract}

D. R. Webb $(\bowtie) \cdot$ R. Silverman • B. Srinivasan • P. S. Lacy

B. Williams · M. J. Davies

Diabetes and Vascular Research Group,

Department of Cardiovascular Sciences, University of Leicester,

Leicester LE1 5WW, UK

e-mail: david.webb@uhl-tr.nhs.uk

K. Khunti $\cdot$ L. J. Gray

Department of Health Sciences, University of Leicester,

Leicester, UK to assess independent metabolic associations with arterial stiffness. Fasting glucose concentration $(\beta=0.10 ; 95 \%$ CI $0.05,0.18 ; p=0.003), 2 \mathrm{~h}$ post-challenge glucose $(\beta=0.14$; $95 \%$ CI $0.02,0.23 ; p<0.001)$ and HOMA-IR $(\beta=0.20$, $95 \%$ CI $0.05,0.53 ; p<0.001)$ were independently related to ${ }_{\mathrm{cf}} \mathrm{PWV}$ after adjustment for age, sex, mean arterial pressure, heart rate, body mass index, renal function and antihypertensive medication.

Conclusions/interpretation IGR characterised by fasting or post-challenge hyperglycaemia is associated with significant vascular stiffening. Post-challenge glucose and HOMA-IR are the most powerful metabolic predictors of arterial stiffness, implying hyperglycaemic excursion and insulin resistance play important roles in the pathogenesis of arteriosclerosis.

Keywords Arterial stiffness · Glucose · Impaired glucose tolerance $\cdot$ Insulin resistance $\cdot$ Pulse wave velocity

\begin{tabular}{ll}
\multicolumn{2}{l}{$\begin{array}{l}\text { Abbreviations } \\
\text { cfPWV }\end{array}$} \\
$\begin{array}{ll}\text { Carotid-femoral pulse wave velocity } \\
\text { CVD }\end{array}$ \\
Cardiovascular disease \\
DM & Newly diagnosed type 2 diabetes mellitus \\
FPG & Fasting plasma glucose \\
HR & Heart rate \\
IFG & Impaired fasting glycaemia \\
IGR & Impaired glucose regulation \\
IGT & Impaired glucose tolerance \\
IR & Insulin resistance \\
MAP & Mean arterial pressure \\
NGM & Normal glucose metabolism \\
2-HPG & Plasma glucose concentration $2 \mathrm{~h}$ after \\
& a 75 g OGTT
\end{tabular}




\section{Introduction}

There is a continuous positive relationship between fasting and post-challenge plasma glucose concentrations and incident cardiovascular events [1]. As the most predictive index of mortality, $2 \mathrm{~h}$ plasma glucose following a standard $75 \mathrm{~g}$ oral glucose tolerance test (2-HPG) may contribute to the pathogenesis of macrovascular disease more than fasting plasma glucose values (FPG) [2].

Premature conduit vessel arteriosclerosis predicts mortality in type 2 diabetes [3] and may be attenuated by therapeutic approaches with potential to lower glucose or blood pressure directly [4-7]. The independent association of FPG and 2-HPG indices with the haemodynamic consequence of early sclerosis (aortic stiffness) implies that modifiable structural mechanisms connect hyperglycaemia with vascular complications even within prediabetes thresholds of impaired glucose tolerance (IGT) [8-11] and impaired fasting glycaemia (IFG) $[12,13]$. These relationships are reported individually [11-13] or as a cumulative effect of the metabolic syndrome. [14-19] and do not specifically address the relative independent strengths of FPG and 2-HPG associations with measures of arterial stiffness.

Establishing the role of insulin resistance in the pathogenesis of thrombotic vascular disease has renewed significance because recent outcome trials using glucoselowering endpoints have failed to prevent cardiovascular events [20-23]. Insulin resistance-mediated premature vascular sclerosis independent of direct glucose lowering may be an important early feature of subclinical atherosclerosis [24-27].

The aim of this study was to compare baseline independent associations of variables of glucose metabolism, namely FPG and 2-HPG concentrations, insulin resistance (HOMA-IR) and $\mathrm{HbA}_{1 \mathrm{c}}$ with carotid-femoral pulse wave velocity ( $\left.{ }_{c f} \mathrm{PWV}\right)$, a validated, non-invasive measure of central aortic stiffness [28]. We sought to characterise premature vascular stiffening in glucose disorders outside the diabetes range and compare the independent contributions of glucose indices to this process within a treatment-naive age- and sex-matched population.

\section{Methods}

Study population We chose a well characterised glucose treatment-naive cohort tagged for vascular outcomes. Volunteers were recruited from a population-based screening programme for diabetes mellitus $[29,30]$. ADDITIONEurope (Anglo-Danish-Dutch Trial of Intensive Treatment in Screen-Detected Diabetes) is a prospective intervention trial of multifactorial cardiovascular risk management in people with screen-detected type 2 diabetes with a primary endpoint of 5 year macrovascular events [31]. As one of the UK arms, ADDITION-Leicester [29, 30] described baseline cardiovascular risk and glucose status by universal $75 \mathrm{~g}$ oral glucose tolerance testing in 6,749 volunteers recruited from 20 general practices between August 2004 and December 2007. ADDITION-Leicester will report macrovascular outcomes for the entire cohort in 2012. Singlesession repeated ${ }_{\text {cf }} \mathrm{PWV}$ measurements were obtained within 4 weeks of a standard $75 \mathrm{~g}$ oral glucose tolerance test, lipid profile, medical history and anthropometric assessment in a subgroup of individuals screened through the ADDITION-Leicester programme. The local research ethics committee approved the study and all participants provided written informed consent.

${ }_{c f} P W V$ measurements Two operators (DRW and RS) blinded to glucose status performed all arterial measurements within a single-site research facility at a university teaching hospital. A standard protocol ensured participants were fasted and rested supine prior to ${ }_{\mathrm{cf}} \mathrm{PWV}$ assessment. Baseline supine brachial artery BP and heart rate (HR) were recorded using a semi-automated oscillometric device (OmronM7, Omron Healthcare, Milton Keynes, UK [Distributor, Medisave (UK) Ltd]). After $10 \mathrm{~min}$ rest, these variables were reassessed every $5 \mathrm{~min}$. ${ }_{\mathrm{cf}} \mathrm{PWV}$ measurements were taken when haemodynamic stability was achieved (defined as two readings within systolic BP of $\pm 9 \mathrm{mmHg}$, diastolic $\mathrm{BP} \pm 6 \mathrm{mmHg}$ and $\mathrm{HR} \pm 8$ beats $/ \mathrm{min}$ [32]. BP and HR concordance was ensured prior to subsequent ${ }_{\text {cf }} \mathrm{PWV}$ measurements. Subjects were supine whilst ${ }_{\text {cf }} \mathrm{PWV}$ was measured using a $4 \mathrm{MHz}$ continuouswave Doppler ultrasound probe (Pulse Trace PT4000; Cardinal Health [formerly known as MicroMedical, Basingstoke, UK]). The cutaneous distance between the site of the femoral pulsation and the sternal notch was repeatedly measured and entered into the device according to manufacturer's instructions [33]. The origin of the pulse waveform was gated to the R-wave of an attached ECG as a timing reference. This enabled quick identification of grossly irregular cardiac rhythms likely to interfere with waveform capture. After archiving a minimum of three $10 \mathrm{~s}$ continuous waveforms, data were processed using established software and mean ${ }_{\mathrm{cf}} \mathrm{PWV}$ was calculated. Captured sequences with waveform ${ }_{\mathrm{cf}} \mathrm{PWV}$ variation greater than $5 \%$ were rejected in line with the manufacturer's recommendations [33]. cf PWV was calculated by dividing the measured surface difference by the respective ECG-derived transit time.

Biomedical measurements Glucose status was determined as fasting and post-challenge (120 min) plasma levels according to WHO criteria [34]. Newly diagnosed type 2 
diabetes mellitus (DM) was defined as either fasting glucose $\geq 7.0 \mathrm{mmol} / 1$ or post-challenge glucose $\geq 11.1 \mathrm{mmol} / 1$ on two occasions at least $48 \mathrm{~h}$ apart. Impaired fasting glycaemia (IFG) was defined as a single fasting glucose concentration between 6.1 and $6.9 \mathrm{mmol} / \mathrm{l}$ and post-challenge glucose $\leq 7.8 \mathrm{mmol} / \mathrm{l}$. Impaired glucose tolerance (IGT) was defined as a single post-challenge glucose concentration between 7.8 and $11.0 \mathrm{mmol} / \mathrm{l}$ and fasting glucose $\leq 6.0 \mathrm{mmol} / \mathrm{l}$. As the aim of the study was to characterise the independent effects of fasting and postchallenge glucose concentrations on ${ }_{\mathrm{cf}} \mathrm{PWV}$, participants with combined intermediate glucose levels (i.e. fasting 6.1$6.9 \mathrm{mmol} / \mathrm{l}$ and post-challenge $7.8-11.1 \mathrm{mmol} / \mathrm{l}$ ) were categorised separately within an IFG/IGT term. Impaired glucose regulation (IGR) was a convenient composite incorporating any defined hyperglycaemia outside the diabetes range (i.e. IFG, IGT and combination IFG/IGT categories). Normal fasting and post-challenge glucose levels were defined by the normal glucose metabolism (NGM) term. Glucose was analysed by the hexokinase method (NADPH production at $340 \mathrm{~nm}$ ) using an Abbott Aeroset system (Abbott laboratories, Maidenhead, UK) and $\mathrm{HbA}_{1 \mathrm{c}}(\%)$ was determined using the Diabetes Control and Complications Trial (DCCT)-aligned Bio-Rad Variant II HPLC system (Bio-Rad laboratories, Hemel Hempstead, UK). Quantitative analysis of serum insulin was carried out by time-resolved fluoroimmunoassay (AutoDelfia 1235; Perkin Elmer LAS, Beaconsfield, UK). The intra-assay $\mathrm{CV}$ for insulin was $<10 \%$. The HOMA-IR calculated score is a well established epidemiological tool validated against direct assessments of insulin sensitivity obtained by euglycaemic glucose clamp techniques [35]. Microalbuminuria was defined as a urine albumin:creatinine ratio in a random sample of more than $2.5 \mathrm{mg} / \mathrm{mmol}$ in men and $3.5 \mathrm{mg} / \mathrm{mmol}$ in women [36]. Estimated mean arterial pressure (MAP) was calculated by adding diastolic and one-third pulse pressure (systolic minus diastolic) measurements recorded during the ${ }_{\mathrm{cf}} \mathrm{PWV}$ session.

Power calculation and statistical analysis The study was powered to determine differences between IGR and DM as well as subgroup categories of IGR. A minimum of 202 (101 per group) participants were required to demonstrate a clinically significant ${ }_{\text {ff }} \mathrm{PWV}$ difference $(>0.8 \mathrm{~m} / \mathrm{s})$ between IGR and DM groups at $80 \%$ power and (two-tailed) $\alpha=$ 0.05 , based on a conservative estimated dependent variable standard deviation of 2.5 [3]. From the data available (using brachial-ankle PWV), 90 (45 per group) participants were required for similar IFG and IGT comparisons ( $80 \%$ power, $\alpha=0.05,{ }_{\text {cf }} \mathrm{PWV} \mathrm{SD}=1.5$ ) [37]. Data were analysed using SPSS software (IBM SPSS ver16, Chicago, IL, USA) and assessed using the Kolmogorov-Smirnov test. Data were checked for normality and appropriate transformations made where necessary. Normally distributed continuous data were compared by ANOVA or Student's independent $t$ test and categorical data were analysed using the $\chi^{2}$ test to determine univariate differences between the cohorts. Adjusted linear regression models were constructed to assess the influence of glucose indices and HOMA-IR as independent predictors of ${ }_{\mathrm{cf}} \mathrm{PWV}$. Variables selected for multivariate regression were based on univariate analysis and factors known to be associated with arterial stiffness (e.g. including classical risk factors for cardiovascular disease [CVD]). Age was added to the model as both a continuous and a grouped variable (quartiles) because of concerns about the non-linear relationship between senescence and ${ }_{c f} \mathrm{PWV}$. The significance level was set at $p<0.05$. Data are presented as mean $\pm \mathrm{SE}$, percentages, counts or ratios, and all tests were two-tailed.

\section{Results}

${ }_{c} P W V$ measurements Overall, $84 \%$ of those participating in ADDITION-Leicester also consented for the arterial measurement substudy. As it was impossible to offer everybody a ${ }_{\text {cf }} \mathrm{PWV}$ appointment within the study timeframe, an eligible population was randomly selected to provide an adequately powered age- and sex-matched sample evenly distributed among the NGM, IGR and DM categories. Operators were blind to this process and participants were specifically asked not to reveal the results of screening prior to the completion of vascular tests. The resulting ${ }_{\mathrm{cf}} \mathrm{PWV}$ measurements were then returned to the independent researcher and matched to glucose data. Ninety-two per cent of waveform sequences were considered of sufficient quality for inclusion, with recognised limitations of the ${ }_{\text {cf }} \mathrm{PWV}$ technique accounting for most of the discounted cases. Thirty-eight participants were excluded because of morphological variability of waveform capture $(n=22)$, electrocardiographic rhythm disturbance (frequent ventricular ectopic activity, extreme HR variability or atrial fibrillation; $n=12$ ) or BP instability $(n=4)$. As there are technical difficulties with waveform acquisition related to BMI, obese (BMI $>30 \mathrm{~kg} / \mathrm{m}^{2}$ ) individuals were unsurprisingly over-represented in the excluded group $(n=15)$. The proportion of DM cases within this group was similar to that in the study population.

Baseline characteristics Glucose comparisons are presented as both categorical (WHO-based [34]) and continuous data. Table 1 describes the biochemical and anthropometric characteristics of the selected NGM ( $n=$ 176), IGT ( $n=142)$, IFG $(n=47)$, IFG/IGT $(n=30)$ and DM $(n=175)$ groups of the ${ }_{c f} \mathrm{PWV}$ study population. As there were no significant differences in baseline characteristics 
Table 1 Demographic and biomedical characteristics of ageand sex-matched ${ }_{c f} \mathrm{PWV}$ study population
Data are $n(\%)$, mean (SD), median (interquartile range) or percentage of participants

${ }^{\text {a }}$ ANOVA (continuous variables) or $\chi^{2}$ test (categorical variables), within-groups trend ${ }^{\mathrm{b}}$ Active prescription of HMGCoA (3-hydroxy-3-methylglutaryl-coenzyme A) reductase or peroxisome proliferatoractivated receptor $\alpha$ inhibitor therapies

${ }^{\mathrm{c}}$ Active prescription of angiotensin-converting enzyme inhibitor, angiotensin receptor blocker, calcium channel blocker or beta blocker therapy

${ }^{\mathrm{d}}$ Composite self-reported history of ischaemic heart disease, cerebrovascular disease or myocardial infarction

\begin{tabular}{|c|c|c|c|c|}
\hline Variable & $\operatorname{NGM}(n=176)$ & IGR $(n=219)$ & $\mathrm{DM}(n=175)$ & $p$ value $^{\mathrm{a}}$ \\
\hline Age (years) & $58.2 \pm 8.9$ & $59.4 \pm 10.4$ & $58.7 \pm 9.8$ & 0.51 \\
\hline Sex ( $\%$ males $)$ & 55 & 53 & 57 & 0.58 \\
\hline Ethnicity (\% WE) & 53 & 57 & 59 & 0.58 \\
\hline FPG (mmol/l) & $5.0 \pm 0.4$ & $5.7 \pm 0.6$ & $7.7 \pm 2.5$ & $<0.01$ \\
\hline 2-HPG (mmol/l) & $5.5 \pm 1.1$ & $8.5 \pm 1.5$ & $13.8 \pm 4.0$ & $<0.01$ \\
\hline $\mathrm{HbA}_{1 \mathrm{c}}(\%)$ & $5.7 \pm 0.4$ & $6.0 \pm 0.5$ & $7.1 \pm 1.5$ & $<0.01$ \\
\hline HOMA-IR (arbitrary units) & $2.07(0.91-2.17)$ & $3.01(1.46-3.70)$ & $4.57(2.48-6.62)$ & $<0.01$ \\
\hline Total cholesterol (mmol/l) & $5.4 \pm 1.1$ & $5.5 \pm 1.1$ & $5.5 \pm 1.3$ & 0.78 \\
\hline HDL-cholesterol (mmol/l) & $1.33 \pm 0.3$ & $1.26 \pm 0.3$ & $1.18 \pm 0.4$ & $<0.01$ \\
\hline Triacylglycerol (mmol/l) & $1.39(0.9-1.6)$ & $1.65(1.0-2.0)$ & $2.20(1.2-2.4)$ & $<0.01$ \\
\hline White cell count $\left(\times 10^{-9} / 1\right)$ & $6.50 \pm 1.71$ & $7.16 \pm 1.64$ & $7.27 \pm 1.64$ & $<0.01$ \\
\hline Alanine transaminase (IU/l) & $21.3 \pm 10.1$ & $24.6 \pm 15.5$ & $30.0 \pm 17.7$ & $<0.01$ \\
\hline Systolic BP (mmHg) & $132.1 \pm 17.0$ & $133.9 \pm 15.3$ & $136.7 \pm 17.9$ & 0.03 \\
\hline Diastolic BP (mmHg) & $80.1 \pm 9.4$ & $81.6 \pm 8.3$ & $82.6 \pm 9.3$ & 0.25 \\
\hline MAP (mmHg) & $98.0 \pm 10.9$ & $99.0 \pm 9.6$ & $100.6 \pm 11.1$ & 0.08 \\
\hline Heart rate (beats/min) & $63.5 \pm 10.3$ & $64.7 \pm 9.9$ & $66.8 \pm 11.1$ & 0.01 \\
\hline Body weight (kg) & $77.7 \pm 15.9$ & $79.8 \pm 15.2$ & $85.8 \pm 17.4$ & $<0.01$ \\
\hline BMI $\left(\mathrm{kg} / \mathrm{m}^{2}\right)$ & $28.2 \pm 4.4$ & $29.0 \pm 4.6$ & $31.1 \pm 5.3$ & $<0.01$ \\
\hline Waist circumference $(\mathrm{cm})$ & $94.7 \pm 12.3$ & $97.3 \pm 11.3$ & $102.8 \pm 11.9$ & $<0.01$ \\
\hline Microalbuminuria (\%) & 4.6 & 10 & 13.3 & 0.03 \\
\hline Lipid-lowering therapy $(\%)^{\mathrm{b}}$ & 20.1 & 20.2 & 21.4 & 0.99 \\
\hline Antihypertensive therapy $(\%)^{\mathrm{c}}$ & 24.7 & 37.4 & 38.2 & 0.01 \\
\hline Active smoking (\%) & 11 & 11 & 10 & 0.35 \\
\hline Cardiovascular disease $(\%)^{\mathrm{d}}$ & 9 & 12 & 11 & 0.08 \\
\hline${ }_{\mathrm{cf}} \mathrm{PWV}(\mathrm{m} / \mathrm{s})$ & $8.91 \pm 1.69$ & $9.70 \pm 2.06$ & $10.04 \pm 2.09$ & $<0.01$ \\
\hline
\end{tabular}

between IGT and IFG, for clarity these are pooled as an IGR $(n=219)$ composite term. These descriptions were comparable to the screened ADDITION-Leicester cohort when grouped using the same diagnostic thresholds (data not presented).

The NGM, IGR and DM groups were well matched, with predictable differences in glucose, lipid and anthropometric variables. Despite more frequent use of antihypertensive therapy brachial systolic pressures were higher in both the IGR and the DM group. There were no significant differences in the nature of the antihypertensive agent prescribed; angiotensin-converting enzyme-inhibitors/angiotensin receptor blockers were used most frequently (57\%), followed by diuretics (43\%), beta blockers $(41 \%)$ and calcium channel antagonists $(28 \% ; p$ for trend $=0.47$, 0.78 and 0.88 for NGM, IGR and DM respectively).

Glucose comparisons Thoraco-abdominal aortic stiffness, as determined by non-invasive ${ }_{\text {cf }} \mathrm{PWV}$, increased with deteriorating glucose metabolism after adjustment for age, MAP, self-reported CVD, sex, BMI, heart rate and antihypertensive therapy. Significant differences were observed between the NGM and IGR categories (adjusted mean $\pm \mathrm{SE}$; ${ }_{\text {cf }} \mathrm{PWV} 9.15 \pm 0.12$ vs $9.76 \pm 0.11 \mathrm{~m} / \mathrm{s}$ respectively, $p \leq 0.001)$ and between the NGM and DM categories ( $9.15 \pm 0.12$ vs DM $9.89 \pm 0.22 \mathrm{~m} / \mathrm{s}$ respectively, $p<0.001$; Table 1, Fig. 1a). This relationship was maintained after stratification of IGR into WHO-defined impaired fasting (IFG, $9.71 \pm 0.12 \mathrm{~m} / \mathrm{s}, p=0.006$ vs NGM) and postchallenge (IGT $9.82 \pm 0.24 \mathrm{~m} / \mathrm{s}, p=0.002$ vs NGM) hyperglycaemic categories. There was no significant difference in mean ${ }_{c f} \mathrm{PWV}$ between IGR and DM (Fig. 1a; $p=0.8$ ). Mean adjusted ${ }_{\mathrm{cf}} \mathrm{PWV}$ was significantly elevated within isolated FPG (FPG abnormality alone $9.77 \pm 0.12 \mathrm{~m} / \mathrm{s}$ vs NGM 9.15 \pm $0.12 \mathrm{~m} / \mathrm{s}, p<0.001)$ and 2-HPG (2-HPG abnormality alone $9.95 \pm 0.22 \mathrm{~m} / \mathrm{s}$ vs NGM $9.15 \pm 0.12 \mathrm{~m} / \mathrm{s}, p<0.001)$ hyperglycaemic categories (Fig. 1b).

Linear regression analyses Univariate analyses were performed to determine the strength of correlations of ${ }_{c f} \mathrm{PWV}$ with FPG, 2-HPG, HOMA-IR and a range of other variables (Table 2). Age $(\beta=0.58, p<0.01), \operatorname{MAP}(\beta=$ $0.52, p<0.01)$, antihypertensive medication $(\beta=0.23, p<$ $0.01)$, self-reported CVD $(\beta=0.21 p<0.01)$, lipid-lowering therapy $(\beta=0.18, p=0.01)$, microalbuminuria $(\beta=0.14$, $p=0.01)$, female sex $(\beta=0.10 p=0.01)$, BMI $(\beta 0.09$, 

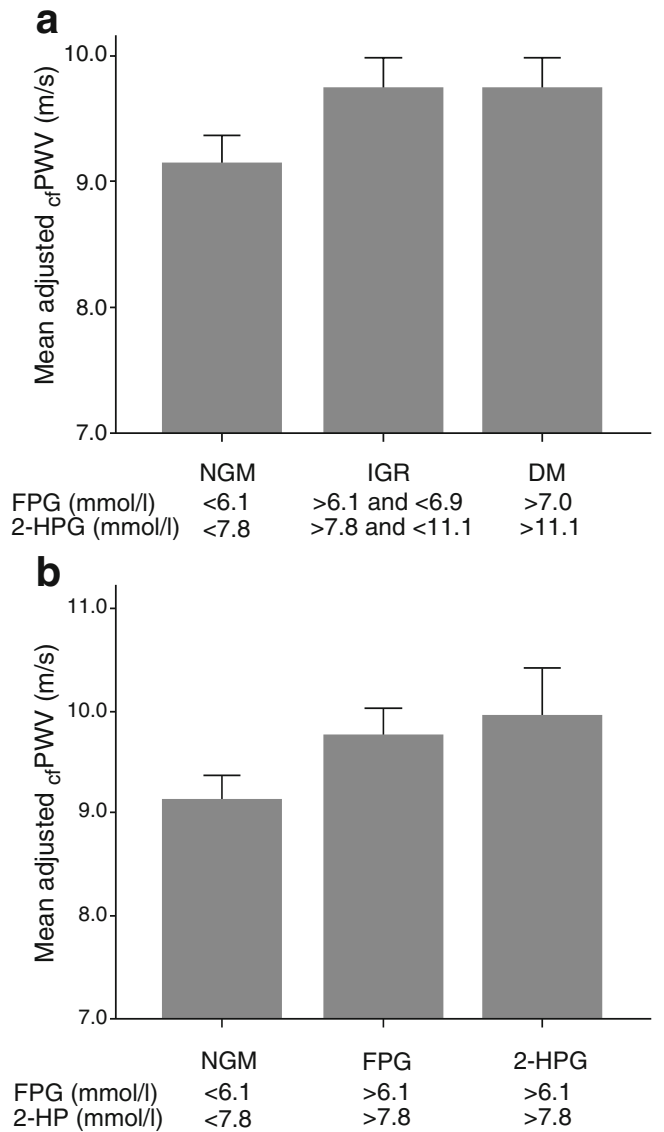

Fig. 1 a Mean adjusted aortic pulse wave velocity $\left({ }_{\mathrm{cf}} \mathrm{PWV}(\mathrm{m} / \mathrm{s})\right.$ and 95\% CI) within WHO normal glucose metabolism (NGM; $n=176$ ), impaired glucose regulation (IGR; $n=219$ ) and diabetes (DM; $n=175$ ) groups. b Mean adjusted aortic pulse wave velocity $\left({ }_{\mathrm{cf}} \mathrm{PWV}(\mathrm{m} / \mathrm{s})\right.$ and $95 \% \mathrm{CI}$ ) within isolated abnormal fasting glucose (FPG; $n=60)$ and $2 \mathrm{~h}$ post challenge glucose (2-HPG; $n=174)$ Mean and $95 \%$ CI of ${ }_{\text {cf }} \mathrm{PWV}(\mathrm{m} / \mathrm{s})$ adjusted for age, MAP, sex and BMI. NGM $(9.15 \pm 0.12)$ vs IGR $(9.76 \pm 0.11), p<0.001$; NGM $(9.15 \pm 0.12)$ vs DM $(9.89 \pm$ $0.22), p<0.001$; NGM $(9.15 \pm 0.12)$ vs abnormal FPG $(9.77 \pm 0.12), p<$ 0.001 ; NGM $(9.15 \pm 0.12)$ vs abnormal $2-\mathrm{HPG}(9.95 \pm 0.22), p<0.001$

$p=0.04)$, FPG $(\beta=0.09, p=0.03), 2-\mathrm{HPG}(\beta=0.15$, $p<0.01)$ and $\log$ HOMA-IR $(\beta=0.14, p<0.01)$ were independently correlated with ${ }_{\mathrm{cf}} \mathrm{PWV}$.

To control the number of covariates entered and limit the effects of collinearity, we constructed three multivariate models to assess the correlations of ${ }_{\mathrm{cf}} \mathrm{PWV}$ with $\mathrm{FPG}, 2-$ HPG, $\mathrm{HbA}_{1 \mathrm{c}}$ and HOMA-IR after adjustment for other factors (Table 3). Model 1 included FPG, 2-HPG glucose or HOMA-IR index, age, MAP, self-reported CVD, sex, BMI, HR and antihypertensive therapy. Lipid-lowering therapy and microalbuminuria were added in place of antihypertensive therapy as independent variables in models 2 and 3 respectively. The strongest positive predictors of ${ }_{c f} \mathrm{PWV}$ in all three multivariate models were age, MAP, sex and HR (standardised $\beta$ coefficients in the FPG analysis were 0.57 , $0.22,0.15$ and 0.15 respectively; all $p \leq 0.01$ ). All three models demonstrated strong relationships between ${ }_{\mathrm{cf}} \mathrm{PWV}$ and FPG (model 1, $\beta=0.09$; model 2, $\beta=0.09$; model 3, $\beta=$ 0.10 ; all $p<0.01$ ), 2-HPG (all three models, $\beta=0.14 p<$ 0.001 ) and HOMA-IR (model 1, $\beta=0.15$; model $2, \beta=0.16$; model 3, $\beta=0.20$; all $p<0.001$ ). The strengths (as measured by standardised $\beta$ coefficients) of 2-HPG and HOMA-IR associations were comparable to those of directly measured haemodynamic covariates (MAP and HR). Multivariate regression within age quartiles made little difference to the contributions of glucose indices to linear and non-linear models (data not shown).

\section{Conclusion}

The results of this study add to the body of evidence advancing premature arteriosclerosis as an important and readily detectable vasculopathic mechanism in latent DM and IGR. Undiagnosed common metabolic diseases that are clinically characterised by fasting or post-challenge hyperglycaemia are often associated with significant thoraco-abdominal aortic stiffness. We found that dichotomising FPG and 2-HPG data into normal and abnormal glucose categories resulted in similar evidence of premature conduit artery stiffness in both isolated fasting and post-challenge hyperglycaemic groups.

Importantly, this study also demonstrates a strong relationship between glucose concentration and arterial stiffening independent of other known risk factors for premature vascular disease. It appears that the structural integrity of the vascular wall is determined by a number of diverse and potentially modifiable pathogenic mechanisms. Impaired glucose regulation, characterised in particular by post-challenge hyperglycaemia and insulin resistance, is a readily treatable example of an alternative pathway promoting large artery stiffness.

All three indices contributed an additional 3-6\% of the variance in ${ }_{c f} \mathrm{PWV}$ after adjustments for age and MAP, although $2 \mathrm{~h}$ post-challenge plasma glucose concentration was found to be a stronger independent predictor of ${ }_{c f} \mathrm{PWV}$ than fasting values. These results imply a dose effect, with a higher mean glucose concentration explaining the more significant contribution of postprandial glucose to ${ }_{\mathrm{cf}} \mathrm{PWV}$ variance. Direct hyperglycaemia-related non-enzymatic glycation of matrix proteins, resulting in the accumulation of glycation end-products within the vessel wall, would provide an explanation for these observations [38, 39]. It is also plausible that insulin resistance syndromes are characterised by abnormalities in the physiological properties of insulin, which normally preserve arterial wave reflections and central haemodynamics [24]. In this population, 2-HPG and HOMA-IR may reflect pro-inflammatory signals induced by visceral fat that alter insulin function and possibly the structural integrity of the vascular wall [40]. We did not attempt to quantify the relative contribution of 
Table 2 Linear regression analysis demonstrating standardised parameter associations in a univariate model with ${ }_{\mathrm{cf}} \mathrm{PWV}$ as dependent variable

\begin{tabular}{llrrr}
\hline Variable & Univariate analysis & & & \\
\cline { 2 - 5 } & $\beta(95 \% \mathrm{CI})$ & Standardised $\beta$ & $p$ value & $R^{2}$ \\
\hline Age & $0.12(0.11,0.14)$ & 0.58 & $<0.01$ & 0.34 \\
MAP & $0.08(0.07,0.09)$ & 0.52 & $<0.01$ & 0.27 \\
Antihypertensive therapy & $0.09(0.66,0.13)$ & 0.23 & $<0.01$ & 0.23 \\
Cardiovascular disease & $1.24(0.67,1.8)$ & 0.21 & $<0.01$ & 0.17 \\
Lipid-lowering therapy & $0.67(0.26,1.09)$ & 0.18 & 0.01 & 0.12 \\
Microalbuminuria & $0.14(0.08,0.19)$ & 0.14 & 0.01 & 0.14 \\
Sex (female) & $0.41(-0.74,-0.07)$ & 0.10 & 0.01 & 0.01 \\
BMI & $0.04(0.01,0.07)$ & 0.09 & 0.04 & 0.01 \\
Heart rate & $0.01(-0.01,0.03)$ & 0.07 & 0.15 & 0.01 \\
Total cholesterol & $0.02(-0.13,0.63)$ & 0.01 & 0.84 & $<0.01$ \\
Active smoking & $0.10(-0.6,0.45)$ & 0.02 & 0.71 & $<0.01$ \\
FPG & $0.10(0.01,0.19)$ & 0.09 & 0.03 & 0.09 \\
2-HPG & $0.08(0.03,0.11)$ & 0.15 & $<0.01$ & 0.14 \\
HbA ${ }_{1 c}$ & $0.13(0.02,0.29)$ & 0.07 & 0.08 & 0.07 \\
Log HOMA-IR & $0.04(0.02,0.11)$ & 0.14 & $<0.01$ & 0.15 \\
\hline
\end{tabular}

wave reflections to elevated ${ }_{\mathrm{cf}} \mathrm{PWV}$, although we recognise that this may be an important determinant of central haemodynamic dysfunction in insulin-resistant states.

The choice of antihypertensive therapy, lipid-lowering or microalbuminuria made little difference to the associations of metabolic factors with arterial stiffness in multivariate analysis, reinforcing the theory that glucose concentration influences ${ }_{\text {cf }} \mathrm{PWV}$ via mechanisms independent of the actions of statins and antihypertensive agents.

As has been reported previously, increased ${ }_{c f} \mathrm{PWV}$ in latent asymptomatic DM or even earlier in the pathogenesis of insulin resistance is an important pathophysiological finding [41]. Implied central artery stiffening and its attendant vascular consequences clearly develop well before conventionally diagnosed diabetes is manifest. Utilising data from prospective studies [3], we estimate that individuals with latent IGR or type 2 diabetes have a 'vascular age' some 5.18 years in advance of chronologi- cally matched counterparts with NGM. Until vascular outcome data are available from randomised controlled trials of screened cases, surrogate indices of atherosclerosis could reliably inform screening strategy. In order to prevent macrovascular complications, our data suggest early identification and intervention in the IGR range is advisable. Mean ${ }_{\text {cf }} \mathrm{PWV}$ in this group was indistinguishable from that in newly diagnosed type 2 diabetes cases. The screening method used will presumably need to reflect the effects that both fasting and post-challenge hyperglycaemia within this range have upon vascular stiffening.

The ADDITION-Leicester ${ }_{\mathrm{cf}} \mathrm{PWV}$ substudy is one of the largest cross-sectional analyses of central artery stiffness conducted within fasting and post-challenge glucoseintolerant states. The study population is tagged for cardiovascular outcome and glycaemic status, which is comprehensively described by universal OGTT testing. Accepted measurements adhere to strict standards; the use

Table 3 Individual glucose/HOMA-IR effects in three multivariate models incorporating combinations of cofactors influencing cf $\mathrm{PWV}$

\begin{tabular}{|c|c|c|c|c|c|c|c|c|c|}
\hline \multirow[t]{2}{*}{ Variable } & \multicolumn{3}{|l|}{ Model $1^{\mathrm{a}}$} & \multicolumn{3}{|l|}{ Model $2^{\mathrm{b}}$} & \multicolumn{3}{|l|}{ Model $3^{\mathrm{c}}$} \\
\hline & $\beta(95 \% \mathrm{CI})$ & $p$ value & $R^{2}$ & $\beta(95 \% \mathrm{CI})$ & $p$ value & $R^{2}$ & $\beta(95 \% \mathrm{CI})$ & $p$ value & $R^{2}$ \\
\hline FPG & $0.09(0.01,0.14)$ & 0.015 & 0.48 & $0.09(0.03,0.17)$ & 0.021 & 0.44 & $0.10(0.05,0.18)$ & 0.018 & 0.48 \\
\hline 2-HPG & $0.14(0.02,0.16)$ & $<0.001$ & 0.51 & $0.14(0.04,0.10)$ & $<0.001$ & 0.46 & $0.14(0.02,0.23)$ & $<0.001$ & 0.51 \\
\hline $\mathrm{HbA}_{1 \mathrm{c}}$ & $0.08(0.05,0.25)$ & 0.007 & 0.48 & $0.10(0.07,0.30)$ & 0.002 & 0.44 & $0.11(0.02,0.28)$ & 0.001 & 0.48 \\
\hline HOMA-IR ${ }_{\log }$ & $0.15(0.05,0.88)$ & $<0.001$ & 0.51 & $0.16(0.04,1.50)$ & $<0.001$ & 0.51 & $0.20(0.05,0.53)$ & $<0.001$ & 0.51 \\
\hline
\end{tabular}

${ }^{a}$ Adjusted for age, MAP, CVD, sex, BMI, heart rate and antihypertensive therapy

${ }^{\mathrm{b}}$ Adjusted for age, MAP, CVD, sex, BMI, heart rate and lipid-lowering therapy

${ }^{\mathrm{c}}$ Adjusted for age, MAP, CVD, sex, BMI, heart rate and microalbuminuria 
of two operators blinded to glucose status minimises variability and ensures robust translation of waveform data. The use of a non-invasive, reproducible gold standard measurement ensures accurate regional representation of central (aortic) stiffness. Detailed phenotyping allows comprehensive regression analyses incorporating a range of covariates influencing ${ }_{\text {ff }} \mathrm{PWV}$. The absence of potentially confounding glucose-lowering therapies in our diabetes group adds strength to the study's aim of assessing the independent effects of glucose on arterial stiffening. An important feature of the study is its population-based approach, introducing clinically relevant ${ }_{\mathrm{cf}} \mathrm{PWV}$ estimates.

Dyslipidaemia, more prevalent within the IGR and NDM categories than elsewhere, did not play an important role in arterial sclerosis, contrasting with the results of the Atherosclerosis Risk in Communities (ARIC) study [26], which reported a synergistic effect of serum glucose and triacylglycerol concentrations on stiffness measures. Statin therapy in particular has been shown to attenuate ${ }_{\text {ff }} \mathrm{PWV}$ and markers of inflammation in diabetic and non-diabetic populations $[6,42]$. It is plausible that hyperlipidaemia and foam-cell-driven plaque formation may affect vascular wall integrity at a later stage in the pathogenic process.

The independent yet relatively minor contributions of glucose indices to ${ }_{\text {cf }} \mathrm{PWV}$ variance correspond with previous studies [14-16] and are predictable given the nature of variables entered into the multivariate analysis. Ageing inevitably dominates determinants of haemodynamic structure and function because of its profound effects on vascular wall integrity, rheology and central aortic pressure. Concerted efforts to recruit a matched study population will have reduced the impact of this variable but it remained necessary to adjust for its effects in multivariate modelling. When the contributions of irreversible determinants of ${ }_{\mathrm{cf}} \mathrm{PWV}$ are considered, potentially modifiable glucose-specific effects become more relevant.

Our study population was relatively young (mean age 59 years) and overtly healthy, which will reduce potential selection bias resulting from the death of older diabetic individuals with particularly stiff arteries, but conversely may make extrapolation of the results to older, higher-risk populations problematic.

Other recognised limitations of the study include potential inaccuracies of waveform acquisition and estimated carotid-femoral distance in overweight individuals. These factors, together with the accepted higher prevalence of ectopic activity, arrhythmia and extreme HR variability in people with type 2 diabetes may have resulted in unavoidable selection bias. The group excluded because of poor waveform concordance did not have a significantly higher waist circumference compared with the study population, suggesting that abdominal obesity per se was not a major confounding influence in ${ }_{c f} \mathrm{PWV}$ acquisition.
Although the method of assessing surface travel distance is important when non-invasively determined ${ }_{\mathrm{cf}} \mathrm{PWV}$ is compared with absolute invasive estimates [43], consistently obtained population comparisons remain valid. We deliberately included BMI in regression modelling despite its clear relationship with diabetes in order to demonstrate the independent effects of glucose indices on ${ }_{\text {ff }} \mathrm{PWV}$. Individuals with unacceptable waveforms attributable to irregular chronotropic activity were evenly distributed across the glycaemic spectrum, and conceivably reflect favourable cardiac function in 'early' diabetes.

Our study was limited by its observational design, which does not enable us to draw conclusions about causal relationships. We are therefore limited to speculating on the importance of cross-sectional independent associations. It is part of the natural course of glucose disorders to develop simultaneous fasting and postprandial hyperglycaemia; this, in combination with a clinical indication to intervene once diabetes thresholds are reached, would make the implementation and interpretation of a prospective comparison extremely difficult. The clearly complex interactions of FPG, 2-HPG and insulin resistance and the demonstrated independent contributions of these indices to subclinical arterial change would probably necessitate a prohibitively large sample size.

These data provide evidence of significant central artery stiffness in impaired glucose metabolism, characterised in particular by post-challenge hyperglycaemia. As measures of arterial compliance have been shown to have prognostic importance in people with established type 2 diabetes, the significance of this relationship in individuals at earlier stages of metabolic disease is clear. Demonstration of prediabetes glucose- and hypertension-mediated macrovascular damage via accelerated senescence is mechanistically plausible and requires further investigation, preferably with intervention studies aimed at ameliorating arterial stiffness. This hypothesis is supported by recent vascular outcome studies in type 2 diabetes implying that glucose lowering may only be effective if introduced early in the natural history of the disease [44]. In this representative glucose treatment-naive population, post-challenge glucose concentration and HOMA-IR were more powerful predictors of arterial stiffness than fasting measures, implying that hyperglycaemic excursion or central obesity-induced insulin resistance are particularly important determinants of vascular wall sclerosis.

Acknowledgements This research was supported by the Novo Nordisk Research Foundation, the NHS Department of Health support for sciences and University Hospitals of Leicester Research and Development internal funding.

Duality of interest The authors declare that there is no duality of interest associated with this manuscript. 


\section{References}

1. Coutinho M, Gerstein HC, Wang Y, Yusuf S (1999) The relationship between glucose and incident cardiovascular events. A meta regression analysis of published data from 20 studies of 95 , 783 individuals followed for 12.4 years. Diabetes Care 22:233-240

2. DECODE Study Group, on behalf of the European Diabetes Epidemiology Group (2001) Glucose tolerance and cardiovascular mortality: comparison of fasting and 2-hour diagnostic criteria. Arch Intern Med 161:397-405

3. Cruickshank K, Riste L, Anderson SG, Wright JS, Dunn G, Gosling RG (2002) Aortic pulse-wave velocity and its relationship to mortality in diabetes and glucose intolerance: an integrated index of vascular function? Circulation 106:2085-2090

4. Satoh N, Ogawa Y, Usui T et al (2003) Anti-atherogenic effect of pioglitazone in type 2 diabetes patients irrespective of the responsiveness to its anti-diabetic effect. Diabetes Care 26:2493-2499

5. Rahman S, Ismail AA, Ismail SB, Naing NN, Abdul Rahman AR (2007) Effect of rosiglitazone/ramipril on preclinical vasculopathy in newly diagnosed, untreated diabetes and IGT patients: 1-year randomised, double-blind, placebo-controlled study. Eur J Clin Pharmacol 63:733-741

6. Dilaveris P, Giannopoulos G, Riga M, Synetos A, Stefanadis C (2007) Beneficial effects of statins on endothelial dysfunction and vascular stiffness. Curr Vasc Pharmacol 5:227-237

7. Nakamura T, Fujii S, Hoshino J et al (2005) Selective angiotensin receptor antagonism with valsartan decreases arterial stiffness independently of blood pressure lowering in hypertensive patients. Hypertens Res 28:937-943

8. Henry RM, Kostense PJ, Spijkerman AM et al (2003) Arterial stiffness increases with deteriorating glucose tolerance status: the Hoorn Study. Circulation 107:2089-2095

9. Schram MT, Henry RM, van Dijk RA et al (2004) Increased central artery stiffness in impaired glucose metabolism and type 2 diabetes: the Hoorn Study. Hypertension 43:176-181

10. Raham S, Ismail AA, Ismail SB, Naing NN, Rahman AR (2008) Early manifestation of macrovasculopathy in newly diagnosed never treated type II diabetic patients with no traditional CVD risk factors. Diabetes Res Clin Pract 80:253-258

11. van Dijk RA, Nijpels G, Twisk JW et al (2000) Change in common carotid artery diameter, distensibility, and compliance in subjects with a recent history of impaired glucose tolerance: a 3-year follow-up study. J Hypertens 18:293-300

12. Ohnishi H, Saitoh S, Takagi S et al (2003) Pulse wave velocity as an indicator of atherosclerosis in impaired fasting glucose: the Tanno and Sobetsu Study. Diabetes Care 26:437-440

13. van Popele NM, Hak EA, Mattace-Raso FU et al (2006) Impaired fasting glucose is associated with increased arterial stiffness in elderly people without diabetes mellitus: the Rotterdam Study. J Am Geriatr Soc 54:397-404

14. Czernichow S, Bertrais S, Blacher J et al (2005) Metabolic syndrome in relation to structure and function of large arteries: a predominant effect of blood pressure - a report from the SU.VI. MAX. Vascular Study. Am J Hypertens 18:1154-1160

15. Ahluwalia N, Drouet L, Ruidavets J et al (2006) Metabolic syndrome is associated with markers of subclinical atherosclerosis in a French population-based sample. Atherosclerosis 186:345353

16. Ferreira I, Boreham CA, Twisk JR et al (2007) Clustering of metabolic syndrome risk factors and arterial stiffness in young adults: the Northern Ireland Young Hearts Project. J Hypertens 25:1009-1020

17. Nakanishi N, Suzuki K, Tatara K (2003) Clustered features of the metabolic syndrome and the risk for increased aortic pulse wave velocity in middle-aged Japanese men. Angiology 54:551-559
18. Schillaci G, Pirro M, Vaudo G et al (2005) Metabolic syndrome is associated with aortic stiffness in untreated essential hypertension. Hypertension 45:1078-1082

19. Safar ME, Thomas F, Blacher J et al (2006) Metabolic syndrome and age-related progression of aortic stiffness. J Am Coll Cardiol 47:72-75

20. The ADVANCE Collaborative (2008) Intensive blood glucose control and vascular outcomes in patients with type 2 diabetes. N Engl J Med 358:2560-2572

21. The Action to Control Cardiovascular Risk in Diabetes Study Group (2008) Effects of intensive glucose lowering in type 2 diabetes. N Engl J Med 358:2545-2559

22. Duckworth W, Abraira C, Moritz T et al (2009) Glucose control and vascular complications in veterans with type 2 diabetes. N Engl J Med 360:129-139

23. Goodarzi MO, Psaty BM (2008) Glucose lowering to control macrovascular disease in type 2 diabetes: treating the wrong surrogate end point? JAMA 300:2051-2053

24. Yki-Jarvinen H, Westerbacka J (2007) Insulin resistance, arterial stiffness and wave reflection. Adv Cardiol 44:252-260

25. Sengstock DM, Vaitkevicius PV, Supiano MA (2005) Arterial stiffness is related to insulin resistance in nondiabetic hypertensive older adults. J Clin Endocrinol Metab 90:2823-2827

26. Salomaa V, Riley W, Kark JD, Nardo C, Folsom AR (1995) Noninsulin-dependent diabetes mellitus and fasting glucose and insulin concentrations are associated with arterial stiffness indexes: the ARIC Study. Circulation 91:1432-1443

27. Van Dijk RAJM, Bakker SJL, Scheffer PG, Heine RJ, Stehouwer CDA (2003) Associations of metabolic variables with arterial stiffness in type 2 diabetes mellitus: focus on insulin sensitivity and postprandial triglyceridaemia. Eur J Clin Invest 33:307-315

28. Laurent S, Cockcroft J, van Bortel L et al (2006) Expert consensus document on arterial stiffness: methodological issues and clinical applications. Eur Heart J 27:2588-2605

29. Davies MJ, Webb DR, Srinivasan B et al (2008) Does screening work for the common good? ADDITION-Leicester: design of a multi-factorial intervention trial in an ethnically diverse population with screen-detected type 2 diabetes. Diabetologia 51(Suppl 1):S426 (Abstract)

30. Webb DR, Khunti K, Srinivasan B et al. (2010) Rationale and design of the ADDITION-Leicester study, a systematic screening programme and randomised controlled trial of multi-factorial cardiovascular risk intervention in people with type 2 diabetes mellitus detected by screening. Trials. doi:10.1186/1745-6215-11-16

31. Sandbaek A, Griffin SJ, Rutten G et al (2008) Stepwise screening for diabetes identifies people with high but modifiable coronary heart disease risk - the ADDITION-Europe Study. Diabetologia 51:1127-1134

32. Mancia G, de Backer G, Dominiczak A et al (2007) 2007 ESHESC practice guidelines for the management of arterial hypertension: ESH-ESC Task Force on the Management of Arterial Hypertension. J Hypertens 25:1751-1762

33. Micro Medical. Pulse Trace PWV (2007). Available from www. micromedical.co.uk/products/pulsetracepwv.asp, accessed 25 January 2010

34. World Health Organization (1999) Definition, diagnosis and classification of diabetes and its complications. A report of a WHO consultation. Part 1: Diagnosis and Classification of Diabetes Mellitus

35. Matthews DR, Hosker JP, Rudenski AS (1985) Homeostasis model assessment: insulin resistance and beta-cell function from fasting plasma glucose and insulin concentrations in man. Diabetologia 28:412-419

36. Lambers Heerspink HJ, Brinkman JW, Bakker SJ, Gansevoort RT, de Zeeuw D (2006) Update on microalbuminuria as a biomarker in renal and cardiovascular disease. Curr Opin Nephrol Hypertens $15: 631-636$ 
37. Morita S, Kasayama S, Otsuki M et al (2006) Atherosclerotic risk factors in Japanese subjects with isolated impaired fasting glucose and those with isolated impaired glucose tolerance according to 1997 and 2003 American Diabetes Association criteria. Diabetes Care 29:2123-2126

38. Wakabayashi I, Masuda H (2006) Association of acute-phase reactants with arterial stiffness in patients with type 2 diabetes mellitus. Clin Chim Acta 365:230-235

39. Aronson D (2003) Cross-linking of glycated collagen in the pathogenesis of arterial and myocardial stiffening of ageing and diabetes. J Hypertens 21:3-12

40. Airaksinen KE, Salela PI, Linnaluoto MK, Ikaheimo MJ, Anola K, Rynanen LJ (1993) Diminished arterial elasticity in diabetes: association with fluorescent advanced glycosylation end products in collagen. Cardiovasc Res 27:942-945
41. Stehouwer CDA, Henry RMA, Ferreira I (2008) Arterial stiffness in diabetes and the metabolic syndrome: a pathway to cardiovascular disease. Diabetologia 51:527-529

42. Jialal I, Miguelino E, Griffen SC, Devaraj S (2007) Concomitant reduction of low-density lipoprotein-cholesterol and biomarkers of inflammation with low-dose simvastatin therapy in patients with type 1 diabetes. J Clin Endocrinol Metab 92: 3136-3140

43. Weber T, Ammer M, Rammer $M$ et al (2009) Non-invasive determination of carotid-femoral pulse wave velocity depends critically on assessment of travel distance: a comparison with invasive measurement. J Hypertens 27:1624-1630

44. Holman RR, Paul SK, Bethel MA, Matthews DR, Neil HAW (2008) 10-year follow-up of intensive glucose control in type 2 diabetes. N Engl J Med 359:1577-1589 